\title{
The BAST algorithm for transit detection
}

\author{
S. Renner ${ }^{1}$, H. Rauer ${ }^{1,2}$, A. Erikson ${ }^{1}$, P. Hedelt ${ }^{1}$, P. Kabath ${ }^{1}$, R. Titz ${ }^{1}$, and H. Voss ${ }^{3}$ \\ ${ }^{1}$ Institut für Planetenforschung, Deutsches Zentrum für Luft- und Raumfahrt (DLR), Rutherfordstraße 2, 12489 Berlin, Germany \\ e-mail: Stefan.Renner@dlr.de \\ 2 Zentrum für Astronomie und Astrophysik, Technische Universität, Hardenbergstr. 36, 10623 Berlin, Germany \\ 3 Departament d'Astronomia i Meteorologia, Facultat de Física, Universitat de Barcelona, Barcelona, C/ Martí Franqués 1, \\ 08028 Barcelona, Spain
}

Received 7 May 2008 / Accepted 26 September 2008

\section{ABSTRACT}

\begin{abstract}
Context. The pioneer space mission for photometric exoplanet searches, CoRoT, steadily monitors about 12000 stars in each of its fields of view. Transit detection algorithms are applied to derive promising planetary candidates, which are then followed-up with ground-based observations.

Aims. We present BAST (Berlin Automatic Search for Transits), a new algorithm for periodic transit detection, and test it on simulated CoRoT data.

Methods. BAST searches for box-shaped signals in normalized, filtered, variability-fitted, and unfolded light curves. A low-pass filter is applied to remove high-frequency signals, and linear fits to subsections of data are subtracted to remove the star's variability. A search for periodicity is then performed in transit events identified above a given detection threshold. Some criteria are defined to better separate planet candidates from binary stars.

Results. From the analysis of simulated CoRoT light curves, we show that the BAST detection performance is similar to that of the Box-fitting Least-Square (BLS) method if the signal-to-noise ratio is high. However, the BAST box search for transits computes 10 times faster than the BLS method. By adding periodic transits to simulated CoRoT data, we show that the minimum periodic depth detectable with BAST is a linearly increasing function of the noise level. For low-noise light curves, the detection limit corresponds to a transit depth $d \simeq 0.01 \%$, i.e. a planet of 1 Earth radius around a solar-type star.
\end{abstract}

Key words. stars: planetary systems - methods: data analysis - techniques: photometric - occultations

\section{Introduction}

The CoRoT satellite (Baglin et al. 2006), successfully launched in December 2006, is the first space-based photometric experiment aimed at discovering extrasolar planets. With a very accurate photometric signal and a continuous time sampling over long periods, it should have the capability of detecting extrasolar planets with sizes down to a couple of Earth radii (Bordé et al. 2003), and will observe a total of 60000 stars in 5 long runs of 150 days each. The first of these long observing runs was performed between May and October 2007, and the scientific analysis of the data is now ongoing. The first detections of transiting planets observed by CoRoT have already been reported (Barge et al. 2008; Alonso et al. 2008; Bouchy et al. 2008; Aigrain et al. 2008; Moutou et al. 2008).

Transit-detection algorithms are used to derive planetary candidates from the analysis of the stellar light curves. Several methods were recently tested with simulated CoRoT light curves (Moutou et al. 2005, 2007). For instance, image processing techniques (Guis \& Barge 2005), removals of low-frequency harmonics, and iterative non linear filters (Aigrain \& Irwin 2004) were used to remove the stellar variability. The transit-search methods included a simple correlation with a sliding transit template, matched filters (Jenkins et al. 1996; Bordé et al. 2007), and several box-shaped search methods such as the Box-fitting Least-Square (BLS) algorithm (Kovács et al. 2002). A theoretical comparison of these detection methods was proposed (Tingley 2003a,b), which concluded that no detector is clearly superior for all transit signal energies, but an optimized BLS algorithm still performs slightly better for shallower transits.

In this paper we present BAST (Berlin Automatic Search for Transits), a new algorithm for periodic transit detection. Using a subset of simulated CoRoT data from Moutou et al. (2005, 2007), we compare its performance with another box-shaped search technique, the BLS algorithm (Kovács et al. 2002). By adding periodic transit events to the light curves, we assess the BAST detection capability with respect to the increase in the noise level.

\section{The BAST algorithm}

Basically, BAST searches for box-shaped signals in normalized, filtered, variability-fitted, and unfolded light curves. It is designed to detect periodic transit events and is based on the preliminary search routine for transits used by the team at DLR (German Aerospace Center, Berlin) in the first blind-test analysis (Moutou et al. 2005). BAST, a sun goddess in Egyptian mythology, is here an acronym for Berlin Automatic Search for Transits. In the following sections, we describe the successive steps, illustrated in Fig. 1, of the BAST algorithm. The parameters used are adapted to the CoRoT mission, for which the temporal sampling of the light curves is $512 \mathrm{~s}$ with a total duration of 150 days. Nevertheless, the algorithm could easily be applied to future space-based transit detection surveys like Kepler or PLATO. 

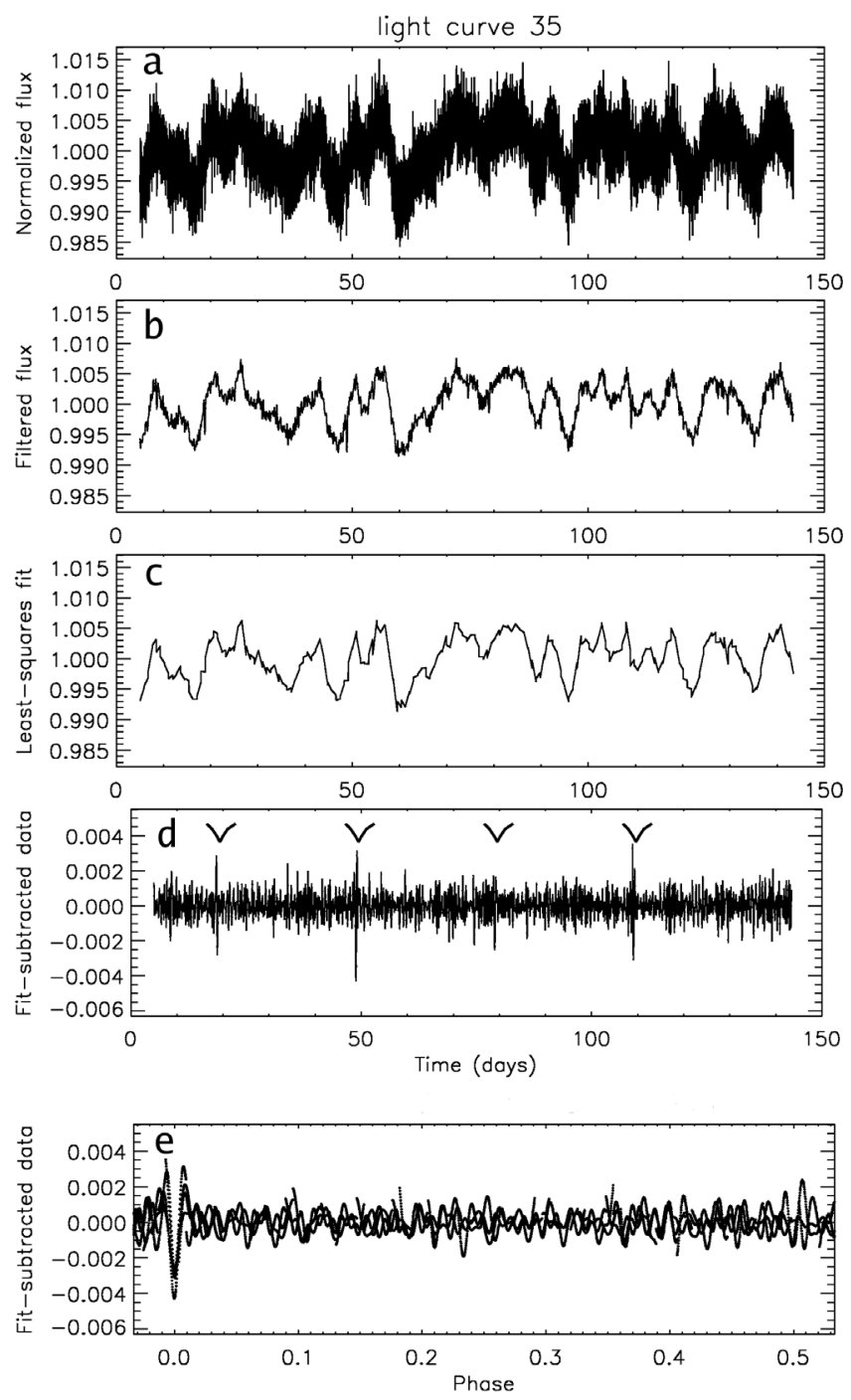

Fig. 1. The successive steps in the BAST algorithm, illustrated with the analysis of a white light curve from the second CoRoT blind test (Moutou et al. 2007). The target is number 35 in the database. From top to bottom: a) the light curve is normalized; b) a low-pass filter is applied to remove high-frequency signals; $\mathbf{c}$ ) the stellar variability is modeled by least-squares fitting to subsections of data; $\mathbf{d}$ ) the fit is subtracted from the filtered light curve, and a box search for periodic transits is performed. The periodic signal is indicated with arrows on the plot; e) the final low-pass filtered, variability-fitted light curve is phase-folded.

\subsection{Light-curve filtering}

First, the light curves are normalized (Fig. 1a). Then, a standard low-pass filter is used to eliminate high-frequency signals (Fig. 1b). The filtering is performed in the frequency domain using the fast Fourier transform. All the frequencies higher than a given cut-off value are removed from the original signal. The dominant periodic signal in the CoRoT light curves is at a period of $\simeq 1.68 \mathrm{~h}$, corresponding to the orbital period. The cut-off period is then varied between $\simeq 1.5$ and $7 \mathrm{~h}$ for the filtering, with a step size of $\simeq 0.3 \mathrm{~h}$. Since one does not expect to detect transiting planets with periods of only a few hours, this value of $7 \mathrm{~h}$ is acceptable. The variation of the cut-off period allows the highfrequency noise to be removed at different levels.

The shape of the transit signals is moderately deformed by this kind of filtering, but the influence of this effect is negligible for the purpose of a detection tool. Another side-effect of the low-pass filtering is that an additional modulation of the light curves occurs at the beginning and the end of the data. Therefore the first and last few days of data (typically between 1 and 10 days) are excluded from the transit search.

\subsection{Stellar variability subtraction}

The stellar variability is fitted locally (Fig. 1c). The light curves are separated into subsections of equal lengths and a linear leastsquares fit to the data is performed for every subsection. The number of subsections is varied from 250 to 20, with a step size of 5 , to model the variability at different scales. For a light curve of 150 days, the size of the subsections is then between $\simeq 0.5$ and 7.5 days. This range of values ensures that no transit events are significantly altered. In a subsequent step, the fit is subtracted from the data (Fig. 1d).

\subsection{Box search for transits}

We compute the standard deviation $\sigma$ of the normalized, lowpass filtered, and variability-fitted light curves. Then a box search for transit-like events is carried out. All data points deviating from the average signal by $k \times \sigma$ are identified, where $k$ is a variable parameter starting at a value of $\simeq 2$. The neighboring deviating points are combined into one single detection. More precisely, consecutive dips separated by less than $\simeq 20 \mathrm{~h}$ are replaced by their mean value. Dips corresponding to transit lengths well below $1 \mathrm{~h}$ are excluded. The resulting mean epochs are listed. Thereafter, the times of the transit-like events found are automatically searched for periodicity: time differences between all detected events are estimated and retained when a single time difference has occurred more than twice within a given error margin. This margin is fixed at 0.1 days. The variable parameter $k \geq 2$ used to identify deviating points increases by values of 0.1 until a periodic signal (or no dips at all) is found.

We point out that this method should allow us to also detect transits when the transit times vary, e.g. due to gravitationally interacting planets.

\subsection{Criteria for identifying planetary candidates}

Once the period has been determined, we estimate the depth and the duration of the transits from the low-pass filtered and variability-fitted light curves. The depth is defined as the mean of the minimum values of all the transit events. For each dip, we estimate the center and the length of the transit. The duration is then defined as the mean of all the segments determined.

Following Seager \& Mallén-Ornelas (2003) and using Kepler's third law, the transit duration $t_{\mathrm{T}}$ for a circular orbit with an impact parameter $b=0$ is given by

$t_{\mathrm{T}} \simeq \frac{P R_{*}}{\pi a}=1.8 R_{*}\left(\frac{P}{M_{*}}\right)^{1 / 3}$,

where $t_{\mathrm{T}}$ is in hours, $R_{*}$ and $M_{*}$ are respectively the radius and the mass of the star in solar units, $P$ is the planet's period in days, and $a$ its semi-major axis. We compute $K=t_{\mathrm{T}} / P^{1 / 3}$, and use the star information to flag transit candidates for which $K$ is much greater than $1.8 R_{*} / M_{*}^{1 / 3}$. Too long a measured transit duration could indeed be due to a problem with the transit parameters, such as an incorrect period. It could also arise from the star parameters, hinting at the possibility of a background eclipsing binary with a spectral type different than that of the target. However, this criterion must be used with care, since there 
are eccentric planets (even at short periods), which can lead to longer transit durations. On the other hand, candidates with very short transit durations (below $1 \mathrm{~h}$ ) are rejected. A condition on the transit depth $(d>5-10 \%)$ is also set to identify obvious eclipsing binaries.

A strong indicator for distinguishing planets from binary stars is the presence of a secondary eclipse in the light curve. Therefore, the phase-folded light curves of the transiting candidates are scanned for shallower transit events, in particular at half of the candidate's period. A phase-folding at twice of the period derived is also performed to detect possible small depth differences.

Modulations can appear in the baseline of the light curves at twice the orbital frequency, which are caused by the tidal forces exerted by a massive stellar companion. Moreover, V-shape transits indicate binaries, too. These phenomena (ellipsoidal variability and V-shape transits) are visually inspected. Because grazing planetary transits also produce $\mathrm{V}$-shape signals, let us note that candidates should not be rejected based on the light curve shape alone.

A significant part of the CoRoT targets are observed in three spatially-separated colors (red, green, blue, but not corresponding to defined photometric systems). Because only planets produce the same transits in the three channels, the transit chromaticity can also be an indicator of binaries. However, the rejection of planet candidates based on the $R, G, B$ colors must be made with caution, since a background star in one of the channels may dilute the planetary transit and then mimic a chromatic effect. We do not apply automatic chromatic criteria. We instead choose to visually check candidates in the 3 color channels, and identify those showing a certain level of chromaticity.

\subsection{Summary of BAST}

To sum up, the BAST algorithm runs as follows:

- The filtering and the fitting of the stellar variability are applied to each light curve (Sects. 2.1 and 2.2), exploring a range of parameter values (cut-off period and size of the fitted subsections).

- A transit search is made (Sect. 2.3) for each cut-off period value and each size of the fitted subsections, and periodic transits found above the detection threshold are stored.

- The transit parameters (period, depth, duration) are calculated, and the possible different detected periods are sorted on the period values.

- Automatic checks are then performed for the transit duration, transit depth, depth differences, and the presence of a secondary eclipse (Sect. 2.4). If several periodic signals remain (such as period multiples), the correct one is then easily identified by eye.

- Finally, the shape of the transits and possible ellipsoidal modulations are visually checked.

A detailed example is given in Fig. 1, with the analysis of a simulated CoRoT white light curve (number 35) from the second CoRoT blind test exercise (Moutou et al. 2007). A transiting planet of 1 Jupiter radius in orbit with a period of 30.1 days around an $\mathrm{A} 5 \mathrm{~V}$ star (corresponding to a depth of $0.3 \%$ ) was added to the light curve. The different steps in the BAST algorithm are displayed, the periodic transits are found, and the transit parameters are retrieved. The algorithm parameters used for this transit detection were: a cut-off period value of $6.25 \mathrm{~h}$, a size of the subsections of $0.9 \mathrm{~d}$, and $3.4 \sigma$ to identify dips deviating from the average signal. The parameters retrieved from the filtered, fit-subtracted light curve are: period $P=30.11 \mathrm{~d}$, depth $d=0.35 \%$, and duration $t_{\mathrm{T}}=5.2 \mathrm{~h}$. On the phase-folded light curve, we can notice again that the shape of the transits is slightly deformed by the filtering. A more detailed analysis of the original light curves can provide accurate transit parameters once a candidate is found. However, this is beyond the scope of this paper. An interesting related work that reconstructs a transit signal in the presence of stellar variability has recently been proposed (Alapini \& Aigrain 2008).

\section{Application to simulated CoRoT light curves}

Two blind detection tests were conducted before the arrival of CoRoT data. In both cases, a set of light curves was generated, including noise sources, stellar variability models (Lanza et al. 2004; Aigrain et al. 2004), and a model of the CoRoT instrument. Then, simulated planetary transit events and a range of contaminating events that mimic transits (eclipsing binaries, variable stars) were added to the light curves.

The first blind test (BT1) aimed at a comparison of the light curve analysis methods to assess the detection performance. A large sample of 1000 light curves was generated with few added transit-like events. The results were published in Moutou et al. (2005).

The primary goal of the second exercise (BT2) was to test the ability to characterize detected transits, and to distinguish transiting planets from eclipsing binaries, using the information from the 3 color channels of CoRoT (Moutou et al. 2007). Thus, 237 light curves in red, green, and blue were built, and all of them contained transits either from planets or from binaries.

Although no detector is clearly superior, the BT1 allowed to conclude that the most sensitive analysis is performed with periodic box-shaped detection algorithms, such as the BLS algorithm (Kovács et al. 2002). This result also appears in a theoretical comparison proposed by Tingley $(2003 a, b)$. Different approaches to analyse the light curves are nevertheless important, since it was also shown that the generation of false positives was method-dependent.

\subsection{BAST applied to BT2 data}

Here, we search transits in the BT2 white light curves with the BAST algorithm. We only use the first 100 light curves (ID 0-99) that are produced assuming a good correction of the residual light scattered from the Earth. For the real CoRoT data, this source of noise is corrected with the reduction pipeline.

We compare the results by also running the BLS algorithm with the same filtering and detrending techniques as BAST (Sects. 2.1 and 2.2). The BLS parameters used are the following (Kovács et al. 2002): $n_{f}=6000$ frequency points in which the spectrum is computed, $m=200$ bins to divide the folded time series, $f_{\min }=0.01$ and $f_{\max }=1 \mathrm{day}^{-1}$ for the minimum and maximum frequencies, $q_{\min }=0.01$ and $q_{\max }=0.05$ for the minimum and maximum fractional transit lengths.

The light curves $0-99$ of the BT2 all contain a transit event, and 12 planets from 0.1 to 1 Jupiter radius are included in 10 of them. The results of the transit search with BAST and with the BLS algorithm are given in Table 1. Both with the BAST and BLS methods, $85 \%$ of the detections are correct, or else affected by a factor of 2 in the period estimation $(P / 2$ or $2 P)$, with respect to the nominal period of the simulated system. More precisely, BAST identifies 76 correct periods, and 9 detections are wrong by a factor of 2 . The BLS method only finds 44 correct periods, and 40 are affected by a factor of 2 with respect to the real 


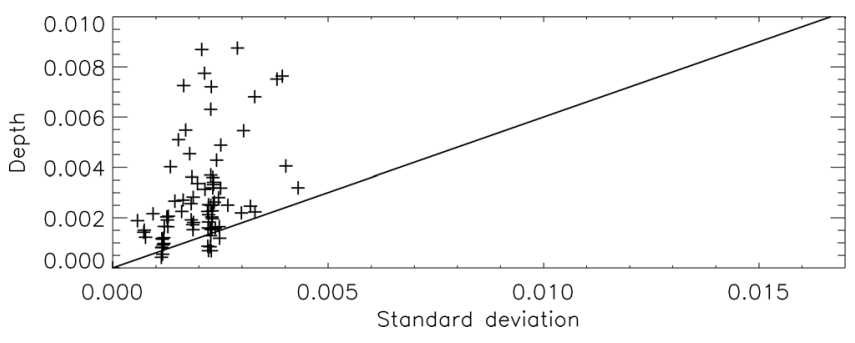

Fig. 2. Depth versus standard deviation for the BT2 light curves 0-99. The line is the BAST detection limit, see Sect. 3.2.

Table 1. BT2 results from the analysis of the (white) light curves 0-99.

\begin{tabular}{ccc}
\hline \hline & BAST & BLS \\
\hline correct $P$ or $P / 2$ or $2 P$ & 76 & 44 \\
$P / 2$ or $2 P$ & 9 & 40 \\
wrong period & 12 & 16 \\
no detection & 3 & 0 \\
planets identified & 4 & 4 \\
\hline
\end{tabular}

period. The eclipsing binaries with similar transits are probably responsible for such confusions.

Note that we have also performed the BT1 exercise again with BAST and BLS, and we get similar results to those in Moutou et al. (2005).

The similar performances obtained here for BAST and BLS stem from the noise level of the BT2 light curves being relatively low. The standard deviation range of the normalized light curves is $0.00057 \leq \sigma_{\mathrm{LC}} \leq 0.00430$ for transit depths $d \leq 1 \%$, see Fig. 2. However, the box transit search of BAST is approximately 10 times faster than the BLS method (with the parameters given in the previous section).

\subsection{Detection capability versus noise}

Here we show how the BAST algorithm behaves with increasing noise level. We selected a subset of 250 BT1 light curves (not containing transit events) with various $\mathrm{S} / \mathrm{N}$; more precisely, the standard deviation range of the normalized light curves of this subset is $0.00039 \leq \sigma_{\mathrm{LC}} \leq 0.01640$. We added simulated periodic transit events to the data, with a fixed duration $t_{\mathrm{T}}=3.5 \mathrm{~h}$ and a fixed period $P=3.689857 \mathrm{~d}$. Then, we slowly decreased the depth to derive the minimum detected signal for given standard deviations. Changing the duration and period values would yield the same results (see the method to identify periodic transits in Sect. 2.3). For few cases, we also performed the same exercise using the BLS algorithm, with the same filtering/detrending techniques as BAST and the same parameters as in Sect. 3.1.

We find that the BAST detection limit is a linearly increasing function of the noise level, with a slope of 0.6. The results are shown in Fig. 3. For each point in the figure, the standard deviation given is an average based on the values of a greater number of light curves, all with the same minimum depths. This was done to give a clear overview of the general trend. We notice that the BLS method is clearly more sensitive than BAST as the noise increases. Nevertheless, BAST can detect periodic signals down to a transit depth $d=0.01 \%$ (i.e. Earth-type planets) for low standard deviation values less than $\sigma_{\mathrm{LC}} \sim 0.001$. Moreover, Fig. 3 shows that comparable or smaller planets than Neptune ( $d \leq \sim 0.3 \%$, depending on the type of the star) are detectable with BAST if $\sigma_{\mathrm{LC}} \leq \sim 0.005$. About $40 \%$ of the monochromatic

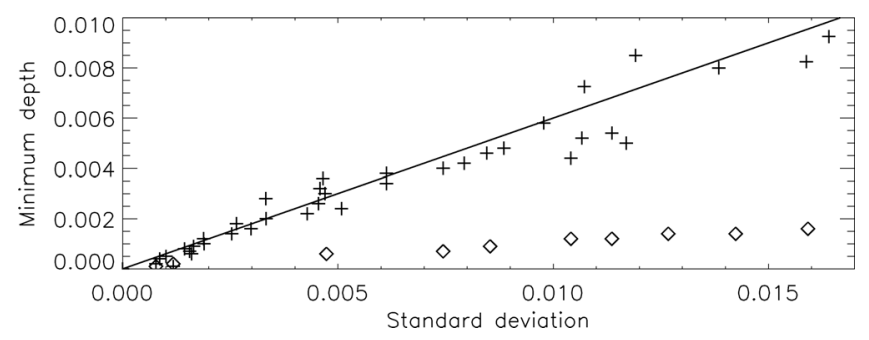

Fig. 3. Minimum depth versus light-curve standard deviation. The plus and diamond signs correspond to BT1 light curves analyzed with BAST and BLS, respectively. A linear fit of slope 0.6 has been included.

light curves of the first CoRoT observing run (IRa01) are within this limit.

\section{Conclusions and prospects}

We have developed BAST (Berlin Automatic Search for Transits), a box-shaped search algorithm to detect transits and identify planetary candidates. From the analysis of BT2 light curves (with relatively high $\mathrm{S} / \mathrm{N}$ ), we have shown that BAST and the BLS transit search method, combined with the same lowpass filtering and linear-fit detrending, perform similarly. The BAST transit search is 10 times faster than the BLS method; indeed, transits are simply identified as individual events deviating from the average signal and then checked for periodicity. By adding simulated periodic transit events to BT1 light curves, we showed that the BAST detection limit is a linearly increasing function of the light curve noise level. Nevertheless, BAST can detect small transiting planets down to the size of the Earth if the $\mathrm{S} / \mathrm{N}$ is sufficiently high. A detailed analysis of the detection capabilities of the various algorithms using real CoRoT data will be published in a future article. Such a study should take the removal of systematic errors into account (Tamuz et al. 2005; Kovács et al. 2005). More work is needed to include these corrections in BAST, which would in turn help to push the detection limit further. Finally, we note that BAST should be sensitive to transiting planets with varying transit times (due to additional perturbing bodies), since it is working on unfolded data and includes a tolerance for period variations.

\section{References}

Aigrain, S., \& Irwin, M. 2004, MNRAS, 350, 331

Aigrain, S., Favata, F., \& Gilmore, G. 2004, A\&A, 414, 1139

Aigrain, S., Collier Cameron, A., Ollivier, M., et al. 2008, A\&A, 488, 43

Alapini, A., \& Aigrain, S. 2008, Proc. IAU Symp., 249

Alonso, R., Auvergne, M., Baglin, A., et al. 2008, A\&A, 482, 21

Baglin, A., Auvergne, M., Boisnard, L., et al. 2006, 36th COSPAR Scientific Assembly, 36, 3749

Barge, P., Baglin, A., Auvergne, M., et al. 2008, A\&A, 482, 17

Bordé, P., Rouan, D., \& Léger, A. 2003, A\&A, 405, 1137

Bordé, P., Fressin, F., Ollivier, M., et al. 2007, ASPC, 366, 145B

Bouchy, F., Queloz, D., Deleuil, M., et al. 2008, A\&A, 482, 25

Guis, V., \& Barge, P. 2005, PASP, 117, 160

Jenkins, J., Doyle, L., \& Cullers, D. 1996, Icarus, 119, 244

Kovács, G., Zucker, S., \& Mazeh, T. 2002, A\&A, 391, 369

Kovács, G., Bakos, G., \& Noyes, R. 2005, MNRAS, 356, 557

Lanza, A. F., Rodonò, M., \& Pagano, I. 2004, A\&A, 425, 707

Moutou, C., Pont, F., Barge, P., et al. 2005, A\&A, 437, 355

Moutou, C., Aigrain, S., Almenara, J., et al. 2007, ASPC, 366, 127M

Moutou, C., Bruntt, H., Guillot, T., et al. 2008, A\&A, 488, 47

Seager, S., \& Mallén-Ornelas, G. 2003, ApJ, 585, 1038

Tamuz, O., Mazeh, T., \& Zucker, S. 2005, MNRAS, 356, 1466

Tingley, B. 2003, A\&A, 403, 329

Tingley, B. 2003, A\&A, 408, 5 